\title{
Identifying XMM-Newton observations affected by solar wind charge exchange. Part I $I^{\star}$
}

\author{
J. A. Carter and S. Sembay
}

Department of Physics and Astronomy, University of Leicester, Leicester LE1 1RH, UK

e-mail: [jac48;sfs5]@star.le.ac.uk

Received 17 April 2008 / Accepted 19 July 2008

\begin{abstract}
Aims. We describe a method for identifying XMM-Newton observations that have been affected by solar wind charge exchange (SWCX) emission and present preliminary results of previously unidentified cases of such emission within the XMM-Newton Science Archive.

Methods. The method is based on detecting temporal variability in the diffuse X-ray background. We judge the variability of a low energy band, taken to represent the key indicators of charge exchange emission. We compare this to the variability of a continuum band, which is expected to be non-varying, even in the case when SWCX enhancement has occurred.

Results. We discuss previously published results with SWCX contamination that have been tested with the above method. We present a selection of observations that we consider to show previously unpublished SWCX enhancements, and further investigate these observations for correlation with data from the solar wind observatory, ACE. We also consider the geometry and viewing angle of XMM-Newton at the time of the observation to examine the origin of the charge exchange emission, whether it be from interactions with geocoronal neutrals in Earth's magnetosheath or from the heliosphere and heliopause.
\end{abstract}

Key words. X-rays: diffuse background - methods: data analysis - Sun: solar-terrestrial relations

\section{Introduction}

This paper is the first of two articles which describe a new method developed to search for instances of solar wind charge exchange (SWCX) contamination of XMM-Newton observations. In this paper we outline the method and provide an initial sample of results. In Paper II we will present the results of applying this method to all suitable observations within the public XMM-Newton Science Archive (XSA).

In this introduction we briefly discuss the SWCX process including examples where this effect occurs in the solar system, and then describe the XMM-Newton observatory and how its orbit affects the likelihood of observing such a process. We describe the Advanced Composition Explorer (ACE) satellite which we use to monitor the solar wind at times of interest.

In Sect. 2 of the paper we detail the steps employed to identify observations possibly affected by SWCX emission and in Sect. 3 we present the results from the use of this method for an initial small sample of approximately 170 observations. We conclude by discussing our plans for the application of this method to incorporate data from the entire publicly available XSA from launch until the commencement of this second phase. As this time period will incorporate more than seven years of XMM-Newton operations, we hope to detect any relationship between our results and the solar cycle.

* Based on observations with XMM-Newton, an ESA Science Mission with instruments and contributions directly funded by ESA Member States and the USA (NASA).

\subsection{Solar wind charge exchange}

SWCX occurs when an ion in the solar wind interacts with a neutral atom and gains an electron in an excited state (Eq. (1)). If the ion is in a sufficiently high charge state in the subsequent relaxation of the ion, an X-ray or UV photon is released (Eq. (2)).

$A^{q+}+B \rightarrow A^{(q-1)^{*}}+B^{+}$

$A^{(q-1)^{*}} \rightarrow A^{(q-1)}+h v$

$A$ is the solar wind ion, for example oxygen, and $B$ the neutral atom, commonly hydrogen or helium in the heliosphere or just hydrogen in the case of geocoronal neutrals in the Earth's magnetosheath.

A SWCX spectrum is characterised by emission lines corresponding to the ion species present in the solar wind. Although the solar wind is approximately $99 \%$ protons, electrons and $\alpha$-particles, the remainder of the wind consists of heavier elements such as $\mathrm{C}, \mathrm{O}, \mathrm{Mg}, \mathrm{Si}, \mathrm{Fe}$ and $\mathrm{Ni}$. The heavier elements found in the solar wind are often of high charge state due to the high temperatures of the solar corona. The relative abundances of the elements are dependent on the conditions of the Sun when the solar wind leaves the solar corona. In this project we concentrate our search on oxygen emission lines as these are typically the strongest SWCX lines in previously published SWCX-enhanced XMM-Newton observations, e.g. Snowden et al. (2004).

SWCX occurs at several locations in the solar system including cometary tails (Greenwood et al. 2000; Cravens 1997), at the 
heliospheric boundary (Cravens 2000; Koutroumpa et al. 2007), planetary atmospheres (Dennerl 2002; Branduardi-Raymont et al. 2007) and from the Earth's magnetosheath or interplanetary neutrals (Cravens et al. 2001; Wargelin et al. 2004; Snowden et al. 2004; Fujimoto et al. 2007). We concentrate on charge exchange between the solar wind and geocoronals in the Earth's magnetosheath and contributions within the heliosphere in the vicinity of the Earth, as this X-ray emission is the most likely to affect the observations of XMM-Newton in terms of a short, temporally varying background source. Local X-ray intensities vary on much shorter timescales (on the order of hours) than those of the heliospheric-ISM boundary contributions, which vary on much longer timescales and with less variation in intensity Robertson \& Cravens (2003b). The main variation seen in the heliospheric contribution occurs in conjunction with changes in the line of sight. As the solar wind travels at approximately $\frac{1}{4} \mathrm{AU}$ per day, the heliospheric emission is the result of the average conditions of the solar wind over several months.

Robertson \& Cravens (2003a), Robertson \& Cravens (2003b) and Robertson et al. (2006) have modelled the expected $\mathrm{X}$-ray emission from charge exchange with geocoronal neutrals, using Hodges (1994) model of the exosphere to account for the densities of the geocoronal neutrals with height for differing solar wind conditions, mapping the area of strongest X-ray emission on the sunward side of the magnetosheath (at an intensity of approximately $8 \mathrm{keV} \mathrm{cm} \mathrm{cm}^{-1} \mathrm{sr}^{-1}$ for nominal solar wind conditions, rising to $200 \mathrm{keV} \mathrm{cm} \mathrm{cm}^{-1} \mathrm{sr}^{-1}$ for simulated solar storm conditions). The solar wind speed can vary dramtically depending on storm or slow wind conditions. A slow solar wind of approximately $300 \mathrm{~km} \mathrm{~s}^{-1}$ and average solar wind densities results in a nominal solar proton flux of approximately $2 \times 10^{8} \mathrm{~cm}^{-2} \mathrm{~s}^{-1}$. The exact distances from the Earth to the magnetopause (the boundary layer between the solar wind plasma and the plasma of the Earth's magnetosphere) and bow shock (which together define the magnetosheath region) depend on the strength of the solar wind at any given time (Khan \& Cowley 1999) but are on average at approximately 9 and $15 R_{\mathrm{E}}$ respectively.

SWCX was first suggested as an explanation for the temporal variations of a few hours to a few days length in the diffuse emission data of the ROSAT All-Sky Survey (long-term enhancements, Snowden et al. 1995) by Cox (1998) and Cravens (2000). SWCX has been seen by XMM-Newton, Chandra and Suzaku, and has been attributed to geocoronal emission (e.g. Fujimoto et al. 2007). Multiple observations with the same pointing direction of the Hubble Deep Field North region taken with XMM-Newton have shown temporal variations in count rates and line emission that has been correlated to enhancements in the level of the solar wind flux and the solar storm of May 2001 (Snowden et al. 2004). Also, depending on viewing geometry, it would be possible to look along the front of a coronal mass ejection (CME) density enhancement, which could cause short term and extreme variations in the level of X-ray flux observed (Collier et al. 2005).

\subsection{The XMM-Newton observatory and viewing geometry}

The XMM-Newton observatory (Jansen et al. 2001) has been used extensively in the study of extended and diffuse X-ray sources. For such studies, a good understanding of the X-ray background is required, including the SWCX, which may affect a user's observation.

Due to the supposed short term variations in the SWCX at the Earth's magnetosheath an observation may be partly or

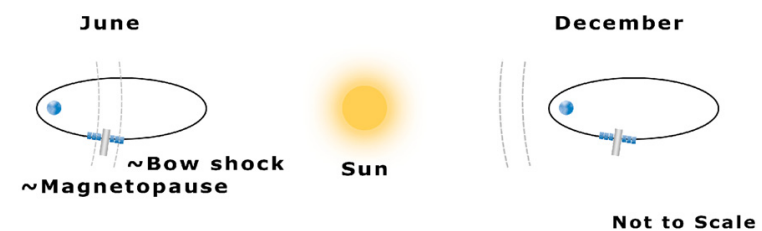

Fig. 1. A schematic illustratration of the orbit of XMM-Newton in relation to the magnetopause and bow shock (dotted lines), which define the boundaries of the magnetosheath, at different times during the year. The left image shows the highly elliptical orbit of XMM-Newton during mid summer (northern hemisphere), and the right image shows the winter configuration. The Sun has been added to show the direction of the Sun and therefore incoming solar wind.

wholly affected by enhancements at energies characteristic of the SWCX line emission. The effect is also of interest in its own right as a monitor of the ionic composition of the solar wind and the interaction between the Earth's magnetosheath and the bombardment of the solar wind.

XMM-Newton is not able to study the SWCX at all times due to the configuration of it's highly elongated elliptical orbit. Optimum viewing of the magnetosheath and areas of the expected highest X-ray emission within the magnetosheath (Robertson \& Cravens 2003b) is expected to occur at only certain points during the $48 \mathrm{~h}$ orbit at certain times of the year, see Fig. 1. The situation is further complicated by restrictions on the viewing angle, which is strongly constrained by the fixed solar panels and those imposed to protect the instruments from directly viewing the Sun and the X-ray and optically bright Earth and Moon, and the non-operational period during the telescope's passage through perigee as it passes through the radiation belts. This results in the observatory being able to view the area of maximum SWCX emission, i.e. the nose of the magnetosheath, only at certain times of the year. XMM-Newton is therefore more likely to experience SWCX-emission enhancement during the summer months (northern hemisphere) as this is when the telescope is able to be in a position to look through the nose and bright flanks of the magnetosheath. However, our goal is to study all observations in the XMM-Newton archive assuming they were conducted in an appropriate observational mode. Coupled with the information regarding the solar wind flux we will, with this project, gain understanding regarding the likelihood and level of SWCX-contamination an XMM-Newton observation may experience.

\subsection{The advanced composition explorer (ACE)}

The Advance Composition Explorer (ACE) was launched in 1997, into orbit at the Earth-Sun Langrangian L1 point about 1.5 million miles from Earth. The instrument package on ACE was designed to study solar, galactic and extragalactic energetic particles (see: http://WWW.srl.caltech.edu/ACE/ ace_mission.html).

In this project we use ACE to provide additional supporting evidence that a SWCX-enhancement may have been seen in an XMM-Newton observation. We use hourly average data (Level 2) from the Solar Wind Electron, Proton, and Alpha Monitor (SWEPAM) instrument on board ACE. Correlations of increased emission in the SWCX lines with increases in the solar wind proton flux lend credence that a particular observation may have experienced a SWCX-enhancement. However the exact scenario maybe complicated by the orientation of the interplanetary magnetic field, adjusting the delay time between ACE 
and Earth (Weimer et al. 2003), so conclusions drawn from correlations between increased X-ray and solar proton flux should be treated with caution. We use data from ACE as a general monitor of the condition of the solar wind flux. However, the delay between ACE and the Earth is approximately one hour.

\section{Method}

In this section we describe the steps taken in the initial data reduction and then continue to detail the method used to test for the presence of SWCX contamination.

The objective when designing the method was to find the key indicators for SWCX-emission, namely short term variability in line emission from the solar wind ion species. Short term variations in the diffuse background indicate a local source, and the presence of line emission at certain energies expected from the solar wind is a diagnostic of SWCX-emission. We grade the indicators and develop a procedure to automatically flag observations that may have experienced SWCX-enhancement throughout their exposure. In this paper we consider single XMM-Newton pointings, but the future paper will explore multiple pointings of the same target to investigate variations between pointings.

\subsection{Data reduction}

The data used in this analysis were taken from the XSA, which can be found at: http://xmm.vilspa.esa.es/external/ xmm_data_acc/xsa/index.shtml.

We used publicly available software in this project, accessible through the web pages of the XMM-Newton Background Working Group (BGWG): http://xmm.vilspa.esa.es/ external/xmm_sw_cal/background/index.shtml.

For each observation an Original Data File (ODF) was downloaded from the XSA. The ODF files were processed using the XMM-Newton Extended Source Analysis Software (ESAS) software package (Kuntz \& Snowden 2008; Snowden et al. 2008), found on the BGWG pages as mentioned above.

Table 1 lists the observations used in this preliminary analysis. We included several observations used by Kuntz \& Snowden (2008) and Snowden et al. (2004) with the aim to use these observations as control subjects for our method. The remainder of the observations were taken from a range of times of year and times in the mission, representive as much as possible of a random selection of observations throughout the XMM-Newton mission.

To undertake our analysis we created lightcurves from event files for each XMM-Newton observation. To do this we filtered the data files for soft proton and particle background contamination using ESAS and other software before commencing with the creation of the lightcurves.

The ESAS software is currently only available for the MOS detectors (Turner et al. 2001) and therefore the analysis detailed here only concerns the MOS instruments. However, improvements to include the PN (Strüder et al. 2001) camera are underway and are expected in an imminent release of the ESAS software. In our future paper we aim to incorporate $\mathrm{PN}$ data into our analysis. Cleaned and calibrated event files were created from the ODF when using the ESAS tool mos-filter. The task mos-filter runs several XMM-Newton Science Analysis Software (SAS) tasks, including emchain for the basic processing of the event files. It then created two lightcurves; one in the field of view and one outside of the field of view from the corners of the detectors for a band between $2.5 \mathrm{keV}$ and $12 \mathrm{keV}$.
Soft proton contamination will fall in the field of view (with the exception of extremely large flares where some soft proton contamination may be scattered into the out of field of view regions), whereas the out of field of view data are only affected by the particle-induced background. A count-rate histogram is then created for the in field of view lightcurve, which should have a roughly Gaussian profile if the observation is unaffected by soft-proton contamination (see the example in Fig. 2). Any large bumps or deviations from the roughly Gaussian shape should be treated with caution as these indicate high levels of contamination. A Gaussian is fitted to the peak of the distribution and using a threshold of $\pm 1.5 \sigma$ this fit is used to create good-time-interval (GTI) periods for the data. The blue vertical lines of Fig. 2 show the range used for the Gaussian fit and the red vertical lines indicate the limits taken for the GTI periods. Further SAS tasks are used to filter the files using the GTI periods to create a cleaned event file. An important point to note is that there may still be residual soft-proton contamination after undergoing these procedures (De Luca \& Molendi 2004).

For each event file we removed sources in the field of view by utilising the source list from the $2 \mathrm{XMM}$ catalogue processing (see http://xmm.esa.int/external/xmm_user_support/ documentation/uhb/node141.html) to create a region file and then extracting the sources from the event file with evselect. We initially used a source extraction radius of 35 arcsec, extending this to remove bright or extended sources if needed.

Checks were made of images created for each cleaned event file to ensure that residual sources were not present. If residual sources remained the extraction radius was increased iteratively until an effectively source-free event file was obtained, although observations with exceptionally bright sources were removed from the sample. The remaining useful observations are shown in Table 1. After this procedure any residual emission from point sources should be negligible. Software for the automated removal of sources will be developed for part II of these articles. We considered observations that use full-frame mode and rejected all other modes for this preliminary study.

We then created spectra and the associated response matrices and auxiliary files for those observations which we wished to study further. We used the ESAS tasks mos-spectra and mosback to create the spectra, background spectra and instrument response and auxiliary files. We used a circular spectrum extraction radius of 17000 detector units to incorporate the in field of view region (pixel size of 0.05 arcsec, 14.2 arcmin radius), centred on DETX, DETY coordinates of $(0,0)$.

\subsection{Examination for SWCX emission}

The first test was to grade the variation in a combined MOS 1 and MOS2 (or with a single instrument if this was the only one available) lightcurve created in a low-energy band covering a SWCX line compared to the variation seen in a lightcurve representing the continuum. The continuum lightcurve covered events in the band $2500 \mathrm{eV}$ to $5000 \mathrm{eV}$. These points were chosen to be in regions where no emission lines would be found. The line emission lightcurve covered events within the $500 \mathrm{eV}$ to $700 \mathrm{eV}$ band, to concentrate on the OVII and OVIII emission at $560 \mathrm{eV}$ and $650 \mathrm{eV}$. For a typical observation, the bands for both line and continuum lightcurves were created so that the total number of counts in each lightcurve were similar. Each lightcurve was created using events with the data flag $F L A G==0$ (those events which are not from a bad pixel, or next to a bad pixel) and the histogram created with a bin size of $1000 \mathrm{~s}$. 
Table 1. Summary of all the observations from the XSA that were used in this analysis. We list the observation identifier, the date of the observation and the exposure of the observation.

\begin{tabular}{|c|c|c|c|c|c|}
\hline Obs. ID. & Exposure (s) & Obs. ID. & Exposure & Obs. ID. & Exposure (s) \\
\hline $00997602010012000-03-22$ & 49332.0 & 0113891001002 2000-04-06 & 11605.6 & 0113891101002 2000-04-07 & 16888.5 \\
\hline $01253001010122000-05-28$ & 32199.8 & $0124711401002 \quad 2000-05-29$ & 17663.9 & $01259202010022000-06-05$ & 23447.8 \\
\hline 0126700401008 2000-06-15 & 7344.45 & $1247104010022000-06-23$ & 8302.49 & 0124711101002 2000-06-24 & 28264.6 \\
\hline $01279210010012000-07-21$ & 53637.1 & $279212010012000-07-23$ & 18456.8 & $01279211010012000-07-23$ & 7264.02 \\
\hline $01277202010012000-07-25$ & 22364.9 & $1125806010012000-07-26$ & 33567.5 & $01112102010012000-07-28$ & 5.45 \\
\hline 0101440101001 2000-09-05 & 49196.7 & 101440701002 2000-09-05 & 29643.4 & 0105460301002 2000-09-07 & 18773.5 \\
\hline 0104460401002 2000-10-12 & 898.8 & $052602010012000-10-13$ & 17851.8 & $01237203010032000-10-27$ & 56029.2 \\
\hline $01114001010012000-11-03$ & 50115.7 & $064601010012000-11-06$ & 54873.2 & $01014404010012000-11-07$ & 45359.8 \\
\hline 0101040301001 2000-11-28 & 37070.4 & $0935527010012001-01-28$ & 24138.1 & 0086360401005 2001-03-12 & 44171.7 \\
\hline $649401010012001-03-24$ & 5378.6 & 83250101004 2001-04-09 & 19805.2 & 0112870201002 2001-04-17 & 16.5 \\
\hline 11550401002 2001-06-01 & 91601.5 & $591402010012001-06-17$ & 12191.9 & $00517601010012001-06-17$ & 7053.58 \\
\hline $0517602010012001-06-18$ & 15740.6 & $1094702010012001-07-11$ & 12481.7 & 0137750101001 2001-07-29 & 17501.8 \\
\hline 0109100201002 2001-08-17 & 935.07 & $001101010012001-08-19$ & 23847.7 & 0089210601001 2001-10-11 & 54619.6 \\
\hline 0089210701001 2001-10-11 & 13630.0 & 89210701002 2001-10-11 & 22315.9 & 0085150201002 2001-10-21 & 37300.7 \\
\hline $851503010032001-10-21$ & 31526.0 & $0227403010012001-11-04$ & 35347.6 & $00287401010012001-11-15$ & 28144.9 \\
\hline 0089210101001 2001-12-01 & & $842302010012001-12-14$ & 28220.5 & $00830003010012001-12-15$ & 26436.8 \\
\hline $01130505010012001-12-23$ & 25154.6 & 084030101001 2001-12-28 & 42853.5 & $00019303010012001-12-28$ & 24582.5 \\
\hline $01068602010012001-12-28$ & 15353.4 & $027403010012001-$ & 6847.05 & 0093200101002 2001-12-29 & 39631.2 \\
\hline 0084140501002 2002-02-04 & 17436.3 & $658201010012002-02-27$ & 49238.9 & $00114202010012002-03-17$ & 587.2 \\
\hline 0110661601002 2002-03-19 & 7267.89 & 058940101002 2002-03-20 & 27788.2 & 0136000101002 2002-04-17 & 17691.4 \\
\hline 0033540601001 2002-05-11 & 5224.01 & $591409010012002-05-22$ & 15889.5 & $00821403010012002-05-22$ & 32887.9 \\
\hline $00943606010012002-05-23$ & 8236.27 & 41750101001 2002-06-15 & 51641.7 & $01345216010112002-06-18$ & 11043.9 \\
\hline 0134521601003 2002-06-18 & 24111.6 & 31740101001 2002-06-19 & 35180.5 & 0135745801001 2002-09-29 & 13120.2 \\
\hline $01129804010012002-09-30$ & 16528.9 & $88502010012002-10-01$ & 23730.5 & $00893705010012002-10-01$ & 54.5 \\
\hline $01475111010012002-10-23$ & & $500501010022002-11-26$ & & $01475118010012002-12-06$ & 90684.3 \\
\hline 0112260801001 2002-12-07 & 13190.8 & $122302010012002-12-18$ & 25541.5 & $0150480501001 \quad 2002-12-22$ & 21904.7 \\
\hline 70340101002 2003-02-02 & 17023.8 & $526808010012003-02-26$ & 16371.9 & $01573606010032003-02-26$ & 8.36 \\
\hline 0146390201001 2003-03-29 & 25388.8 & 0153220601001 2003-05-28 & 10902.0 & $01506801010012003-07-26$ & 42666.2 \\
\hline 0152131201002 2003-08-16 & 9502.71 & $495403010012003-08-17$ & 25734.6 & 0049540401001 2003-08-19 & 24037.2 \\
\hline 0149630301001 2003-09-16 & 19768.3 & 17740401007 2003-10-05 & 27582.9 & 0017740201007 2003-10-09 & 17879.9 \\
\hline 0017740601007 2003-10-11 & 22288.5 & 0017740701007 2003-10-13 & 21736.3 & $01477601010012003-10-14$ & 36842.6 \\
\hline 0017740501007 2003-10-20 & 551.5 & $1487425010012003-10-28$ & 7917.18 & 0162160201002 2003-11-24 & 14003.8 \\
\hline 0162160401002 2003-12-06 & 10509.2 & 162160601002 2003-12-14 & 12360.0 & 0205260201009 2004-01-16 & 13440.0 \\
\hline 0201230201001 2004-01-21 & & $2002403010012004-04-02$ & 15255.5 & $01645607010012004-07-23$ & 8.0 \\
\hline 0164560901001 2004-09-12 & 58437.8 & 0205970201001 2004-09-20 & 48145.6 & 0206060201001 2004-11-03 & 31485.3 \\
\hline 0202610801001 2004-11-09 & 7179.57 & 0201440101001 2004-11-09 & 10311.2 & 0202610801002 2004-11-09 & 17784.4 \\
\hline 0201903101001 2004-11-10 & 27833.5 & $2019005010012004-11-12$ & 27163.9 & $02033609010012004-11-20$ & 27258.5 \\
\hline 0203240201017 2004-12-04 & 18804.6 & 0203240201001 2004-12-05 & 19919.2 & $02033601010012004-12-11$ & 30644.7 \\
\hline $02006302010012004-12-20$ & 44644.7 & 0202370301001 2005-01-08 & 25837.4 & 0202130301001 2005-01-15 & 37385.1 \\
\hline 0212480701002 2005-06-05 & 19008.7 & 0211280101002 2005-06-12 & 42621.5 & $03045315010062005-06-22$ & 15215.0 \\
\hline $03059206010012005-06-23$ & 15224.2 & $03040501010012005-06-30$ & 69689.5 & $02124808010012005-07-01$ & 48830.5 \\
\hline 0302420101001 2005-07-08 & 85607.2 & $03045317010022005-07-10$ & 7502.96 & $01645714010012005-08-21$ & 56486.1 \\
\hline $0300800101002 \quad 2005-08-31$ & 45875.7 & 0300540101001 2005-09-14 & 23398.9 & $03031001010012005-09-26$ & 52629.3 \\
\hline 0300600401001 2005-10-08 & 28648.7 & $03055601010012005-10-21$ & 27595.6 & 0302310501002 2005-10-23 & 23162.3 \\
\hline 0159760301001 2005-11-01 & 37543.4 & $03023516010072005-11-27$ & 43034.0 & $0302310301002 \quad 2005-12-18$ & 43998.8 \\
\hline 0303560201001 2005-12-19 & 6516.42 & & & & \\
\hline
\end{tabular}

Lightcurves were rejected from the sample if they were less than $5000 \mathrm{~s}$ long or if greater than $10 \%$ of the bins had less than 20 counts, for either lightcurve.

The lightcurves were filtered using the previously determined GTI file (as part of the ESAS output), and the counts in each bin adjusted for any reduction in exposure of that bin. Bins that had undergone severe GTI filtering where more than $40 \%$ of the bin was removed were excluded. The Poisson error of each time bin and each lightcurve was computed and adjusted for any reduction in exposure of that bin.

For each set of lightcurves we created line-continuum scatter plots, an example of which is shown in Fig. 3. If no enhancement due to SWCX has occurred, we assumed correlation between the line and continuum bands. Soft protons are spectrally variable in intensity and shape but show no lines. If residual soft proton contamination has occurred (which is most likely to be slowly varying after the significant flare periods have been removed by GTI filtering), the bands chosen for the line and continuum lightcurves are sufficiently close that any spectral change in the continuum band should be reflected in the line band, and therefore this assumption holds. We looked at the scatter plot for several observations that were expected to have experienced high levels of residual soft proton contamination and indeed the straight line approximation was valid. SWCX-enhanced observations were expected to show much less correlation between the line and continuum bands. Bins that experience SWCX-enhancement would show a deviation from the fit with increased count rate levels in the line band.

A fit to each scatter plot is computed using the IDL procedure, linfit, which fits a linear model to a set of data by 

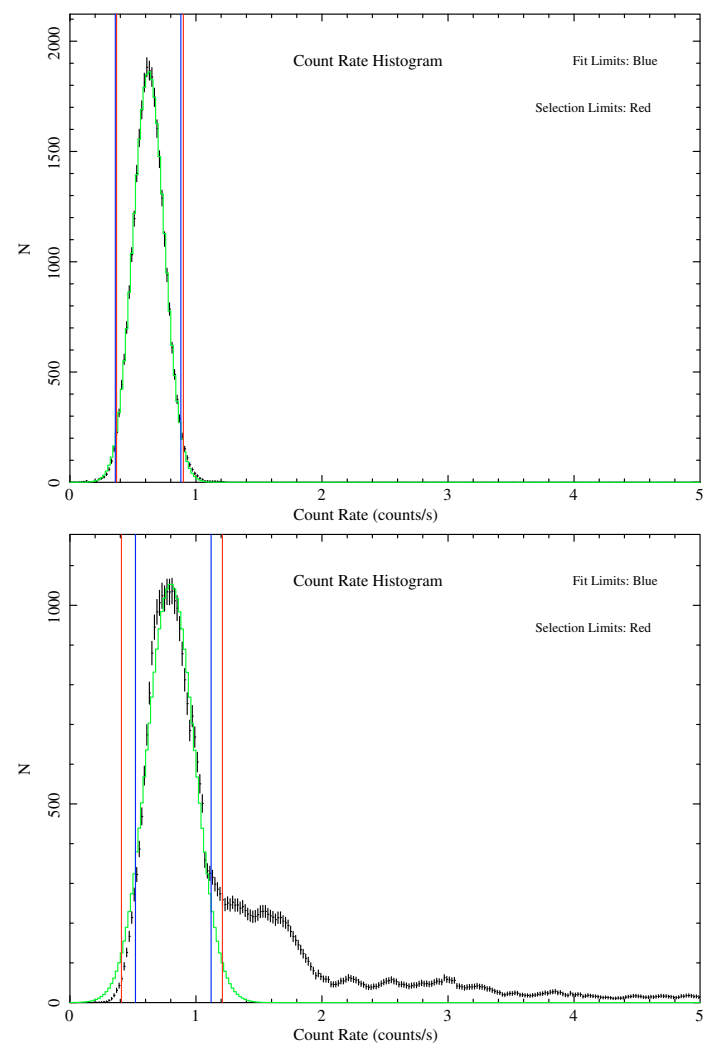

Fig. 2. Example count rate histograms from the ESAS software where a Gaussian shaped curve is fitted to the histogram. The upper panel shows a count rate histogram where the data and the Gaussian curve are almost coincident and little or no flaring has occurred, whereas the lower panel shows flaring characteristics.

minimising the $\chi^{2}$ statistic, see Eq. (3). We use the continuum band count rate as the independent variable and the values of the dependent variable are taken to be the count rate in the line band,

$\chi^{2}=\sum_{i}\left(D_{i}-E\right)^{2} / \sigma_{i}^{2}$

$D$ are the points of the line-continuum scatter plot, $E$ is the expected value, as found by the fit to the line-continuum scatter plot and $\sigma$ the error on each point.

The procedure gives a $\chi^{2}$ for each fit to the scatter plot. We divide this $\chi^{2}$ by the degrees of freedom of the fit to obtain the reduced- $\chi^{2}$ for the fit, hereafter referred to as $\chi_{\mu}^{2}$. A high $\chi_{\mu}^{2}$ indicates that a significant fraction of the points have a large deviation from the best fit line found by the fit, and therefore we expected that these cases would be more likely to show SWCX-enhancement.

In addition we computed the $\chi^{2}$ values for each individual lightcurve and calculate the ratio between the line and continuum band $\chi^{2}$ values to add to our diagnostic (hereafter denoted as $R_{\chi}$ ).

As a measure of possible residual soft proton contamination, we computed the ratio of in field of view to out of field of view counts in a band between $7000 \mathrm{eV}$ and $12000 \mathrm{eV}$, correcting for the area lost due to the removal of point sources. To do this we used a script fin_over_fout which is publicly available through the BGWG web-site (as previously mentioned) where a high ratio (hereafter denoted as Fin/Fout) indicates a large residual soft proton contamination component $(<1.15$ not contaminated, 1.15-1.3 slightly contaminated, 1.3-1.5

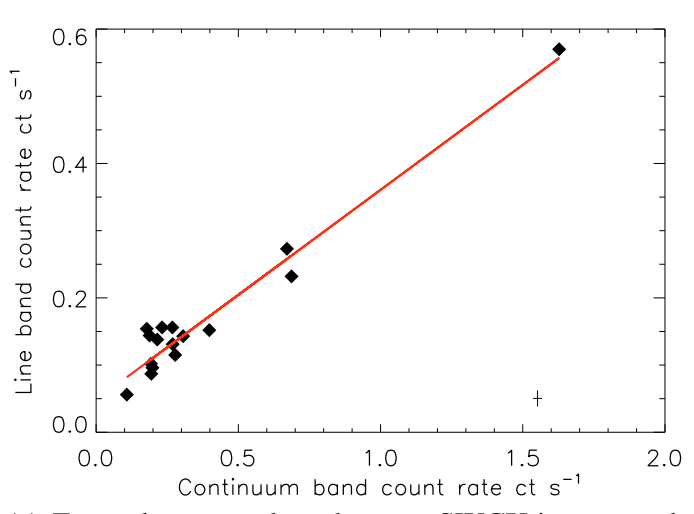

(a) Example scatterplot where no SWCX is expected, showing a good line fit to the scatterplot producing a low $\chi_{\mu}^{2}$ value

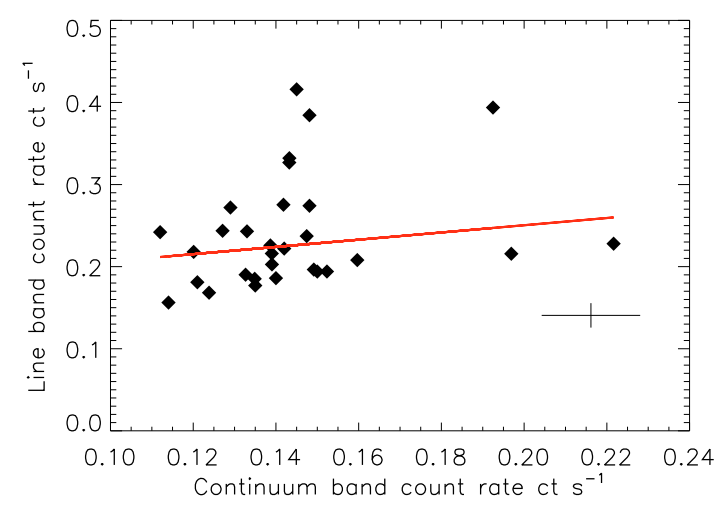

(b) Example scatterplot where SWCX has been observed, showing deviations from the line fit resulting in a high $\chi_{\mu}^{2}$ value

Fig. 3. Example scatter plots between the line and continuum bands for an observation that has experienced high levels of residual soft proton contamination, yet still shows a good linear fit between the line and continuum count rates (first panel) and an observation that shows SWCX-emission features (second panel). Representative average error bars are plotted in the bottom right-hand corner of each plot.

very contaminated, $>1.5$ extremely contaminated). We also considered the background time series files, as produced as part of the 2XMM Serendipitous Source Catalogue (http://xmm. esa.int/external/xmm_user_support/documentation/ uhb/node141.html). These files contain lightcurves for the highest energy events (created for full-field events with PI > $14 \mathrm{keV}$ and with the selection expression (PATTERN==0) \&\& \#XMMEA_22 \&\& ((FLAG \& 0x762ba000) == 0)). Large and/or frequent flaring periods in the time series would suggest that the observation suffered from soft proton contamination or that a residual component may still be present depending on the effectiveness of the flare filtering procedure. Closer scrutiny of the GTI filtering in such a case is required.

\section{Results}

In this section we present the general trends in the results from our sample. We briefly discuss the results from the control subjects that were added to our sample. These were observations with previously detected SWCX-enhancement and have published results. We then discuss several examples of previously unreported cases of SWCX-enhancement, identified by the method described in this paper, and that produced the highest $\chi_{\mu}^{2}$ 


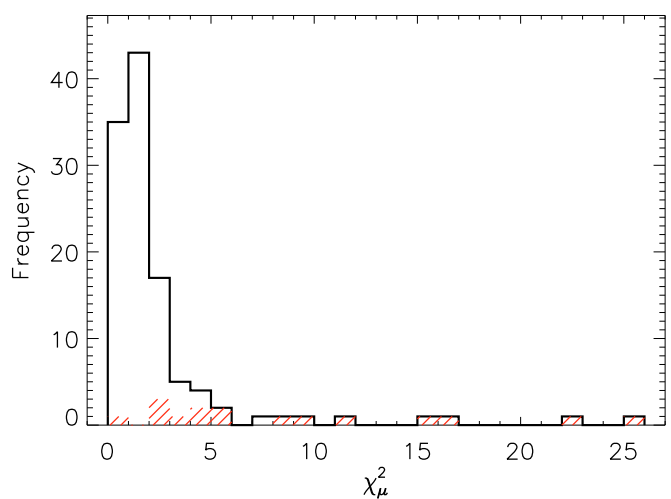

Fig. 4. Histogram of $\chi_{\mu}^{2}$ values.

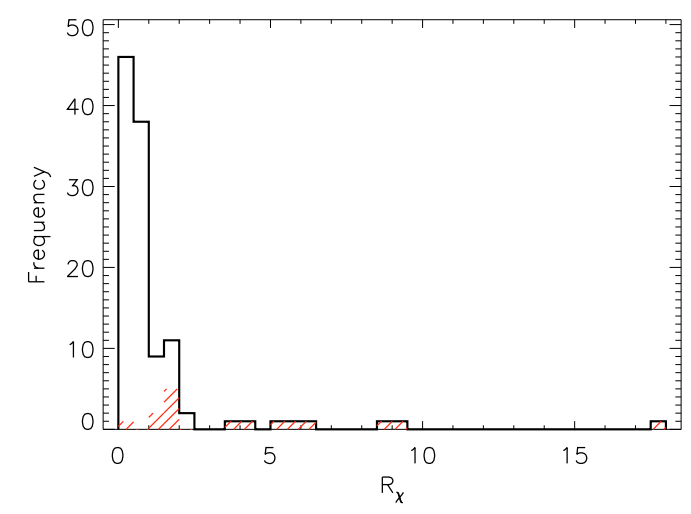

Fig. 5. Histogram of $R_{\chi}$.

to the linear fit. The observations were ranked by $\chi_{\mu}^{2}$ and the top 30, for brevity, are summarised in Table 2. We have removed observations from the sample that showed a significant deviation in the distribution of count rates from the Gaussian that was determined and used by the ESAS software for filtering purposes.

\subsection{General trends}

In Fig. 4 we present a histogram of the $\chi_{\mu}^{2}$ values found for the whole data set. The modal value for the $\chi_{\mu}^{2}$ value is between 1.5 and 2.0. We see that there are several cases with extreme $\chi_{\mu}^{2}$ values, showing the greatest disparity between line and continuum count rates. We expect the SWCX cases to be found with the highest $\chi_{\mu}^{2}$ values. Shaded blocks in all figures in this section indicate those observations with a suspected or previously published SWCX enhancement, as will be discussed in the following sections.

In Fig. 5 we present a histogram of the $R_{\chi}$, which is the ratio between the $\chi^{2}$ value for the line band lightcurve to the $\chi^{2}$ value for the continuum band lightcurve. SWCX-enhancements will have increased variation in the line band compared to the continuum band, so a high $R_{\chi}$ is expected for SWCX-affected observations. The shaded blocks show that there are several observations where high variability is found along with high $R_{\chi}$ values. High SWCX-enhanced observations are expected for these cases.

In Fig. 6 we present a histogram of the Fin/Fout ratios. This shows that twelve observations with a high $\chi_{\mu}^{2}$ value have been judged extremely contaminated by residual soft protons as they show a Fin/Fout ratio $\geq 1.5$. The two vertical dashed lines in this

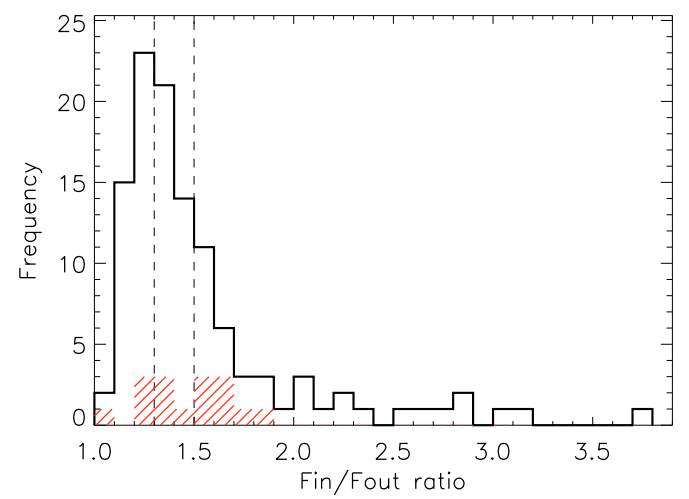

Fig. 6. Histogram of Fin/Fout ratios.

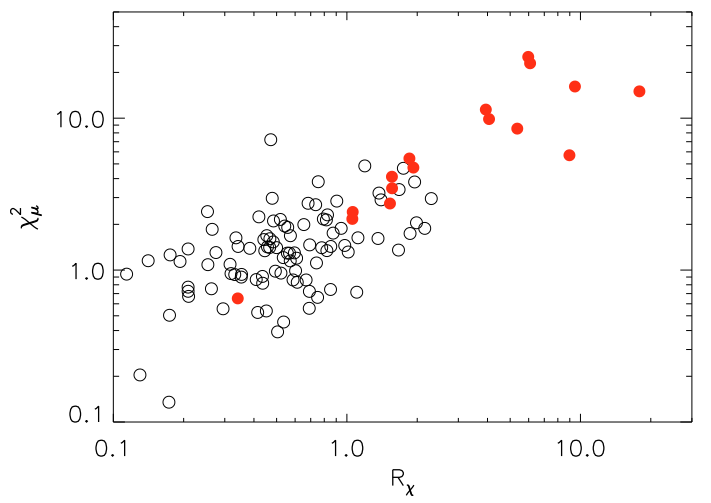

Fig. 7. Scatter plot of $\chi_{\mu}^{2}$ versus $R_{\chi}$ values. Filled circle are used for observations with a suspected or previously published SWCX enhancement.

figure indicate the Fin/Fout thresholds for classification as being very contaminated $(1.3-1.5)$ or extremely contaminated $>1.5$ by residual soft protons.

In Fig. 7 we plot $\chi_{\mu}^{2}$ values versus their corresponding $R_{\chi}$. In general, the highest $R_{\chi}$ are seen to have the highest $\chi_{\mu}^{2}$, as expected for SWCX enhancements. The filled circles, representing those observations with a suspected (this study) or previously published SWCX enhancement, are found with the highest $R_{\chi}$, with one notable exception. These cases are discussed in Sects. 3.2 and 3.3. The exceptional case, where both the $R_{\chi}$ and $\chi_{\mu}^{2}$ are low, corresponds to a SWCX-enhanced control observation that is unidentified by this method, and this case is discussed within Sect. 3.2. The distribution in this figure suggests that one may be able to start to define a threshold, between the parameters $\chi_{\mu}^{2}$ and $R_{\chi}$, whereby a user would suspect that SWCX was extremely likely to have occurred. This will be addressed further in Paper II. In Fig. 8 we plot $R_{\chi}$ values versus their corresponding Fin/Fout values. SWCX-enhanced observations show a range of Fin/Fout values.

\subsection{Cases matching previously identified SWCX-enhanced observations}

Several observations in our sample, used as control subjects, have been previously studied by others and have been identified as having experienced SWCX-enhancement. The Snowden et al. (2004) observation of the Hubble Deep Field North (case $\{7\}$, observation number 0111550401 ) produced a $\chi_{\mu}^{2}$ value of 8.53 and is ranked highly likely in Table 2 . Kuntz \& Snowden (2008) 
Table 2. Results for the observations which showed the highest values of $\chi_{\mu}^{2}$. Each observation is given a case number (column one) and the observation identification number is shown in column two. We give the date, the exposure time (Exp.), the degrees of freedom $(v)$ after any flarefiltering, the $\chi_{\mu}^{2}$ value and then state the ratio between the $\chi^{2}$ value of the line lightcurve and that of the continuum lightcurve $\left(R_{\chi}\right)$. We also state the average Fin/Fout (FF) ratio (and error on this value) between the MOS1 and MOS2. The final column gives a comment about the observation as described in the text.

\begin{tabular}{|c|c|c|c|c|c|c|c|c|c|}
\hline Case & Observation & Date & $\begin{array}{l}\text { Exp. } \\
\text { (ks) }\end{array}$ & $v$ & $\begin{array}{c}\chi_{\mu}^{2} \\
\text { linfit }\end{array}$ & $R_{\chi}$ & $\begin{array}{l}\mathrm{FF} \\
\text { ratio }\end{array}$ & $\begin{array}{l}\text { Err. FF } \\
\text { ratio }\end{array}$ & Comment \\
\hline 1 & 0085150301 & $2001-10-21$ & 31.96 & 25 & 25.31 & 5.98 & 1.806 & 0.09 & Strong case SWCX \\
\hline 2 & 0093552701 & 2001-01-28 & 24.17 & 16 & 22.97 & 6.09 & 1.486 & 0.09 & Weak case SWCX \\
\hline 3 & 0149630301 & 2003-09-16 & 19.77 & 16 & 16.15 & 9.47 & 1.066 & 0.06 & Strong SWCX \\
\hline 4 & 0305920601 & $2005-06-23$ & 15.24 & 14 & 15.01 & 17.77 & 1.226 & 0.07 & Strong SWCX \\
\hline 5 & 0150680101 & 2003-07-26 & 42.67 & 30 & 11.39 & 3.93 & 1.274 & 0.06 & Strong SWCX \\
\hline 6 & 0101040301 & $2000-11-28$ & 37.21 & 35 & 9.86 & 4.06 & 1.655 & 0.07 & Weak case SWCX \\
\hline 7 & 0111550401 & 2001-06-01 & 93.37 & 83 & 8.53 & 5.36 & 1.339 & 0.04 & Snowden et al. (2004) \\
\hline 8 & 0302310501 & $2005-10-23$ & 23.16 & 23 & 7.21 & 0.47 & 2.535 & 0.12 & Low $R_{\chi}$ \\
\hline 9 & 0127921101 & $2000-07-23$ & 7.43 & 6 & 5.70 & 8.97 & 1.310 & 0.12 & Kuntz \& Snowden (2008) \\
\hline 10 & 0070340501 & 2001-06-18 & 19.10 & 8 & 5.43 & 1.85 & 1.760 & 0.14 & Weak case SWCX \\
\hline 11 & 0202370301 & 2005-01-08 & 25.85 & 14 & 4.85 & 1.19 & 1.202 & 0.07 & Low $R_{\chi}$ \\
\hline 12 & 0127921001 & $2000-07-21$ & 54.04 & 53 & 4.73 & 1.93 & 1.660 & 0.07 & Kuntz \& Snowden (2008) \\
\hline 13 & 0202610801 & 2004-11-09 & 17.90 & 15 & 4.68 & 1.75 & 1.378 & 0.08 & No SWCX \\
\hline 14 & 0136000101 & 2002-04-17 & 17.75 & 17 & 4.12 & 1.56 & 1.570 & 0.09 & Strong case SWCX \\
\hline 15 & 0150480501 & $2002-12-22$ & 21.93 & 11 & 3.82 & 0.75 & 1.467 & 0.11 & Low $R_{\chi}$ \\
\hline 16 & 0112580601 & 2000-07-26 & 33.66 & 32 & 3.80 & 1.95 & 2.171 & 0.11 & No SWCX \\
\hline 17 & 0101440101 & 2000-09-05 & 49.22 & 31 & 3.45 & 1.56 & 1.534 & 0.07 & Weak case SWCX \\
\hline 18 & 0305560101 & 2005-10-21 & 23.01 & 22 & 3.39 & 1.67 & 1.755 & 0.09 & No SWCX \\
\hline 19 & 0001930301 & $2001-12-28$ & 24.58 & 18 & 3.21 & 1.37 & 2.010 & 0.10 & No SWCX \\
\hline 20 & 0125920201 & 2000-06-05 & 23.45 & 22 & 2.97 & 0.48 & 1.389 & 0.10 & Low $R_{\chi}$ \\
\hline 21 & 0135745801 & 2002-09-29 & 13.16 & 12 & 2.96 & 2.30 & 1.162 & 0.08 & No SWCX \\
\hline 22 & 0089370501 & 2002-10-01 & 49.23 & 22 & 2.90 & 1.40 & 1.143 & 0.06 & No SWCX \\
\hline 23 & 0113050501 & $2001-12-23$ & 25.64 & 19 & 2.84 & 0.91 & 1.216 & 0.06 & Low $R_{\chi}$ \\
\hline 24 & 0147511101 & $2002-10-23$ & 97.33 & 31 & 2.75 & 0.68 & 1.373 & 0.06 & Low $R_{\chi} \&$ bad flaring \\
\hline 25 & 0164560701 & 2004-07-23 & 31.62 & 20 & 2.74 & 1.53 & 1.520 & 0.07 & Weak case SWCX \\
\hline 26 & 0099760201 & 2000-03-22 & 49.34 & 35 & 2.69 & 0.74 & 1.436 & 0.06 & Low $R_{\chi}$ \\
\hline 27 & 0085150201 & 2001-10-21 & 37.33 & 21 & 2.43 & 0.25 & 1.834 & 0.09 & Low $R_{\chi}$ \\
\hline 28 & 0106460101 & 2000-11-06 & 54.90 & 43 & 2.41 & 1.06 & 1.308 & 0.06 & Weak case SWCX \\
\hline 29 & 0000110101 & 2001-08-19 & 24.00 & 9 & 2.31 & 0.83 & 1.355 & 0.09 & Low $R_{\chi}$ \\
\hline 30 & 0205970201 & 2004-09-20 & 48.14 & 35 & 2.24 & 0.42 & 1.466 & 0.06 & Low $R_{\chi} \&$ bad flaring \\
\hline
\end{tabular}

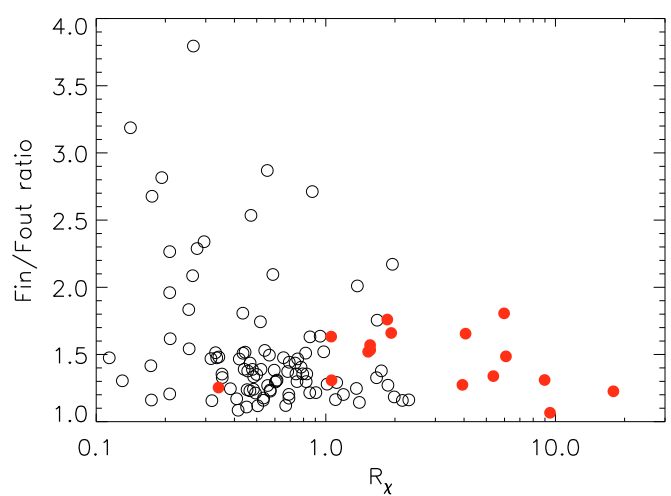

Fig. 8. Scatter plot of $R_{\chi}$ versus Fin/Fout values. Filled circles are used for observations with a suspected or previously published SWCX enhancement.

looked at several observations of the Groth-Westfall Strip. These were (observation numbers 0127921001(GWS1), 0127921101(GWS2) and 0127921201(GWS3)) and the first two were reported to have enhanced OVII and OVIII emission (correlated with enhanced solar wind flux as compared to a low solar proton flux for GWS3). GWS1 (case $\{12\}$ ) and GWS2 (case $\{9\}$ ) had $\chi_{\mu}^{2}$ values of 4.73 and 5.70 whereas GWS3 gives a $\chi_{\mu}^{2}$ value of only 1.2 (case $\{63\}$, not included in Table 2 ). GWS1 is judged to be very contaminated by residual soft protons, as is mentioned in Kuntz \& Snowden (2008) and it showed considerable particle contamination. The lightcurves for the line and continuum band along with the ACE solar proton flux during the observation are plotted in Fig. 9. Scatter plots for these observations are plotted in Fig. 10. An observation of the Polaris Flare region considered in the analysis of Kuntz \& Snowden (2008), which was reported to have a SWCX-enhancement, is ranked as case $\{31\}$ and therefore narrowly misses out on inclusion in Table 2. Other observations discussed by Kuntz \& Snowden (2008) were ranked as unlikely to have experienced a SWCX-enhancement using our criteria, which was in correlation with the diagnosis by Kuntz \& Snowden (2008), when no SWCX-enhancement was found. However, there was one exception to this case where our indicators suggested no SWCX effects had occurred whereas Kuntz \& Snowden (2008) states the contrary (case $\{103\}$, observation number 0162160201). This is an example of a false negative detection by our grading system whereby formally the scatter of the line to continuum band count rates is insignificant and therefore the observation is disregarded. Also, our criteria will not identify observations that are affected by uniform SWCX throughout the entirety of their exposure period, which seems to be the case in this example. It should be noted in this particular case the number of degrees of freedom was not particularly high and on inspection of the lightcurves no obvious SWCX-enhanced period could be easily identified. 


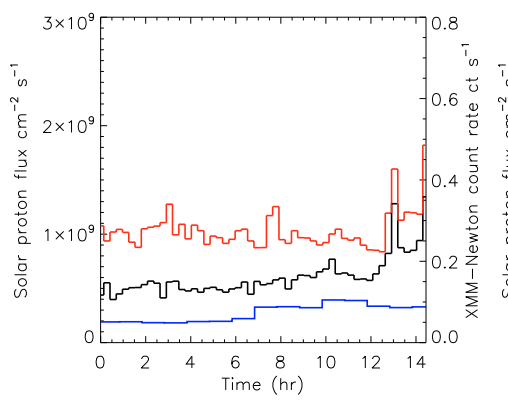

(a) Case $\{12\}$, GWS1

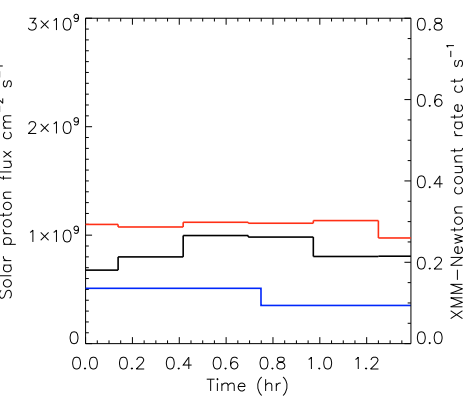

(b) Case. $\{9\}$, GWS2

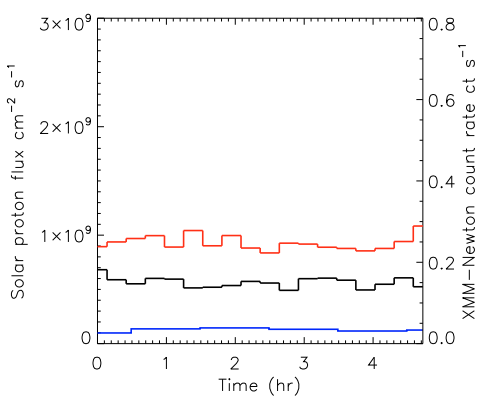

(c) Case. $\{63\}$, GWS3

Fig. 9. Lightcurves for observations of the Groth-Westhall Strip. Blue represents the solar proton flux, black the line band count rate and red the continuum band count rate. These observations were studied spectrally by Kuntz \& Snowden (2008) and cases $\{13\}$ and $\{10\}$ were found to have SWCX-enhancement, whereas $\{64\}$ was devoid of SWCX features.

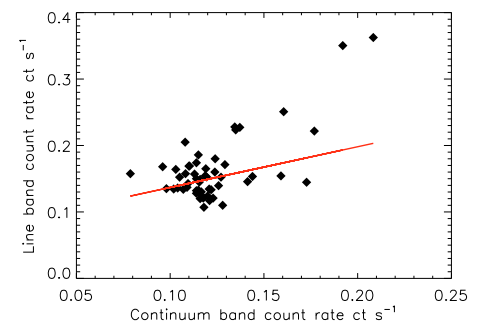

(a) Case $\{12\}$, GWS1

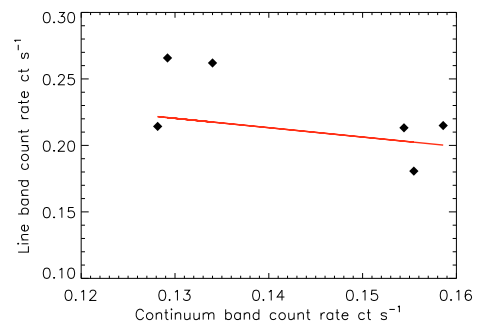

(b) Case $\{9\}$, GWS2

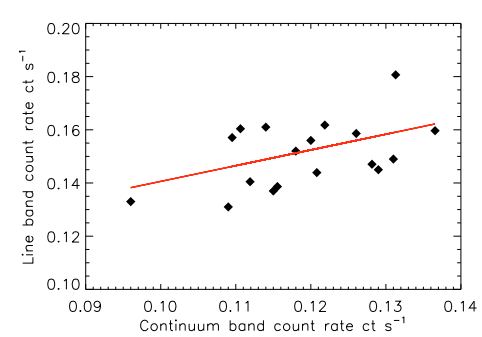

(c) Case $\{63\}$, GWS3

Fig. 10. Scatter plots for observations of the Groth-Westhall Strip. These observations were studied spectrally by Kuntz \& Snowden (2008) and cases $\{13\}$ and $\{10\}$ were found to have SWCX-enhancement, whereas $\{64\}$ was devoid of SWCX features.

\subsection{Cases of newly identified SWCX-enhancement}

As shown in Table 2, there is a large number of observations that show high variability between the line and continuum band and therefore produce a high $\chi_{\mu}^{2}$ value. Index numbers in this section refer to the index numbers of Table 2 . In this section we briefly describe observations with currently unpublished SWCX-enhancements, starting with those cases that have strong indicators. The XMM-Newton lightcurves, orbital positions and spectra for these cases are shown in Fig. 12. We also present several cases where SWCX-enhancements might have occurred and which we classify as weak or dubious cases. The XMM-Newton orbital positions, lightcurves and spectra for these cases are presented in Fig. 13. Orbital positions are plotted in the geocentric solar-ecliptic $X-Y$ plane and the geocentric solar-ecliptic $X-Z$ plane. These plots are designed to aid the visualisation of the line of sight of XMM-Newton during an observation; in particular we wished to test whether the telescope was observing through the zone of the brightest model geocoronal X-ray flux, as shown in Robertson et al. (2006). The dark blue lines show the approximate minimum and maximum positions, at the time of the observation, of the magnetopause and bow shock respectively. At the subsolar point this is calculated using Khan \& Cowley (1999) and the shape of these boundaries have been approximated by a parabola. The path of XMM-Newton during the period of the observation is shown in these plots and is coloured black for the stages of the orbit which corresponds to the period of expected SWCX-enhancement and red otherwise. The dotted lines on these plots represent the line of sight of the telescope and the start and end of the observation.

There are several cases which appear in Table 2 but that are not described below for a number of reasons.

1. The overall variation in both lightcurves was significantly large that a detection of variation in the line curve could not be made, resulting in a high value of $\chi_{\mu}^{2}$ but a low value of $R_{\chi}$ (cases 8,11, 15, 20, 23, 26, 27 and 29).

2. There was no time in the lightcurves when the line band tracked the continuum band meaning that $\chi_{\mu}^{2}$ was still high, so if SWCX was present, it was spread throughout the observation $(13,16,18,19,21$ and 22).

3. There was significant residual soft proton contamination after flare removal, meaning that it was statistically difficult to detect variation unique to the line band (24 and 30).

\subsubsection{Example strong cases of SWCX-enhancement}

\section{- Case: $\{1\} 0085150301$}

This observation shows a good correlation between the line band lightcurve and a peak in the solar proton flux. For the non-enhanced period, the line and continuum bands show the same count rate structure. This is the most extreme case of SWCX-enhancement in our sample and the richest in terms of line emission species observed. The OVIII clearly becomes visible during the enhancement period. In addition, many other emission lines are apparent. Below the oxygen lines, the carbon lines have appeared, but more strikingly clear are the NeIX and MgXI lines, at $0.91 \mathrm{keV}$ and $1.34 \mathrm{keV}$ respectively. There are also emission lines seen between $0.65 \mathrm{keV}$ and $1 \mathrm{keV}$ that could be attributed to OVIII or various species of iron, and an enhancement slightly above the silicon instrumental line at approximately $1.8 \mathrm{keV}$. An increase in continuum level is seen during the SWCX period between $1100 \mathrm{eV}$ and $1275 \mathrm{eV}$. This observation shows considerable residual soft proton contamination, indicated by the high Fin/Fout value, which may explain the elevation of the continuum level. However, this does not explain the increase in ratio between a particular line and the continuum band, which we attribute to SWCX-enhancement. 
Table 3. SWCX line emission seen in the spectrum of case $\{1\}$.

\begin{tabular}{cc}
\hline \hline Ion & Line energy $(\mathrm{keV})$ \\
\hline CVI & 0.37 \\
CVI & 0.46 \\
OVII & 0.56 \\
OVIII & 0.65 \\
OVIII/FeXVII? & 0.81 \\
NeIX & 0.91 \\
NeX/FeXVII? & 1.03 \\
NeIX & 1.15 \\
FeXVII & 1.20 \\
MgXI & 1.34 \\
MgXII/SiXIII? & 1.85 \\
SXIV? & 2.000 \\
\hline
\end{tabular}

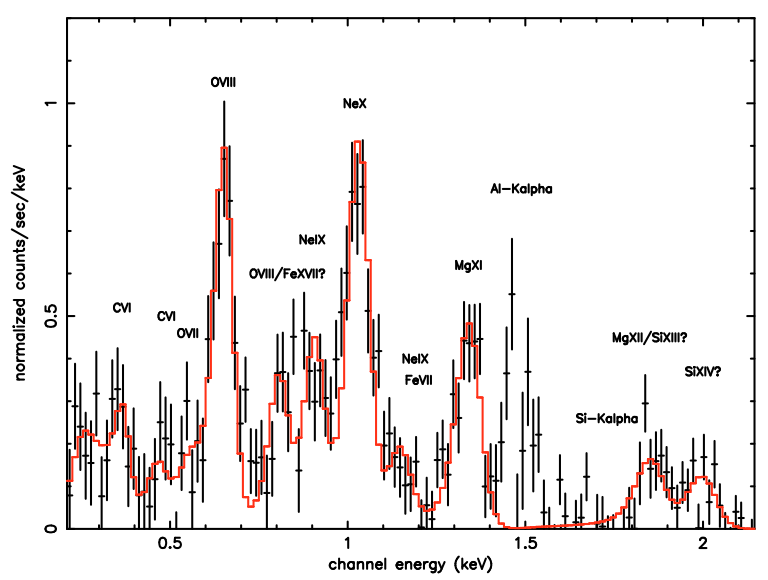

Fig. 11. The resultant spectrum for case $\{1\}$. The model to the SWCX lines folded through the instrument response is plotted in red. The excess seen around $1.49 \mathrm{keV}$ and $1.74 \mathrm{keV}$ results from particleinduced instrumental background.

In Fig. 11 we plot the resultant spectrum, using the non SWCX-enhanced period as the background spectrum. The background has been scaled to the SWCX-enhanced period between $2.1 \mathrm{keV}$ and $2.5 \mathrm{keV}$, to account for residual soft proton contamination throughout the observation. We fit the resulting spectrum with the lines detailed in Table 3, giving the identification of the corresponding ion we consider responsible. It was necessary to add the instrumental fluorescent lines $\mathrm{Al}-\mathrm{K}_{\alpha}$ and $\mathrm{Si}-\mathrm{K}_{\alpha}$ to the fit. This background component is time variable and therefore is not completely accounted for by the scaling between the non-enhanced and SWCX-enhanced period, hence this residual component is still present in the spectrum. We also consider the possibility that this enhancement is linked to the CME event of the 19th October 2001 (Wang et al. 2005). The delay between the occurrence of this event at the solar corona and its arrival near Earth would be approximately three days. This CME was registered by ACE and therefore passed in the relatively near vicinity of the Earth. Such an event could explain the large enhancements and richness of the spectrum, seen for this example of SWCX-enhancement.

- Case: $\{3\} 0149630301$

This observation towards the Large Magellanic Cloud shows a line band over continuum band enhancement in the latter part of the observation. ACE also shows a solar proton flare that rises in the second half of the observation. XMM-Newton is travelling in the nose of the magnetosheath. The enhancement was expected in the latter part of the observation, approximately two hours after the start of the observation. The count rate histogram shows a good Gaussian fit and the Fin/Fout ratio is low. The background time series file however, shows a large flare at the end of the observation that adds some doubt to our diagnosis. The $R_{\chi}$, at 9.47 , is high. The spectrum created for the supposed enhanced period is interesting as it shows low energy enhancement below $0.7 \mathrm{keV}$ along with an apparent line at around $1.35 \mathrm{keV}$ that could be attributed to MgXI.

- Case: $\{4\} 0305920601$

This observation was highly variable in the oxygen band, producing a very high $\chi_{\mu}^{2}$ value of approximately 15 . It had a low value for the Fin/Fout ratio and a good fit to the count rate histogram from the ESAS software. The $R_{\chi}$ was the highest in the sample. This observation was conducted in the summer months, so XMM-Newton was found in the nose of the magnetosheath. The solar wind flux was relatively high throughout the observation, with a peak greater than $1 \times$ $10^{9} \mathrm{~cm}^{-2} \mathrm{~s}^{-1}$. It can be observed from the lightcurve that there is a step at approximately two hours, before which the line band shows much more variability than the continuum band. The enhancement period shows increased flux from the OVIII line and below and possibly some increase around $0.9 \mathrm{keV}$ which could be attributed to NeIX.

- Case: $\{5\} 0150680101$

This observation was conducted in July so that XMM-Newton was positioned in the nose of the magnetosheath. The ACE data are a little sparse, but when available the solar proton flux was high, peaking above $1.5 \times 10^{9} \mathrm{~cm}^{-2} \mathrm{~s}^{-1}$. A time cut was taken at approximately nine hours as a SWCX-enhanced period was expected in the latter half of the observation. The spectrum shows OVII and OVIII enhancements and there is also a strong enhancement seen below $0.5 \mathrm{keV}$ where lines from carbon are expected.

- Case: $\{14\} 0136000101$

The lightcurves for this observation showed a sharp peak in the line band during the last bin. This observation was taken in April when XMM-Newton is in the flanks of the magnetosheath at approximately 90 degrees to the Earth-Sun line. The solar proton flux shows a sudden step to a particularly high level nearing $2 \times 10^{9} \mathrm{~cm}^{-2} \mathrm{~s}^{-1}$. Depending on the delay taken between ACE and the magnetosheath, which will be approximately one hour, this step could correspond to the peak seen in the lightcurves. The Fin/Fout ratio shows this observation to be very contaminated by residual soft protons. This case also shows an increase in flux from the OVIII line and below, although there is no evidence and very poor statistics for SWCX lines with greater energies, such as NeIX and $\mathrm{MgXI}$.

\subsubsection{Example weak cases of SWCX-enhancement}

\section{- Case: $\{2\} 0093552701$}

This observation was very contaminated by soft protons yet shows a high $R_{\chi}$ of 6.09. The combined spectrum for the enhanced period showed an increase in flux for both the OVII and OVIII lines over the non-enhanced spectrum, although it exhibits less lower energy flux around the carbon lines in comparison to the strong cases of SWCX-enhancement. There was little solar proton flux data available during this observation.

\section{- Case: $\{6\} 0101040301$}

This observation shows a very high $R_{\chi}$ of 4.06 and is very contaminated by residual soft protons. The slow rise in the 

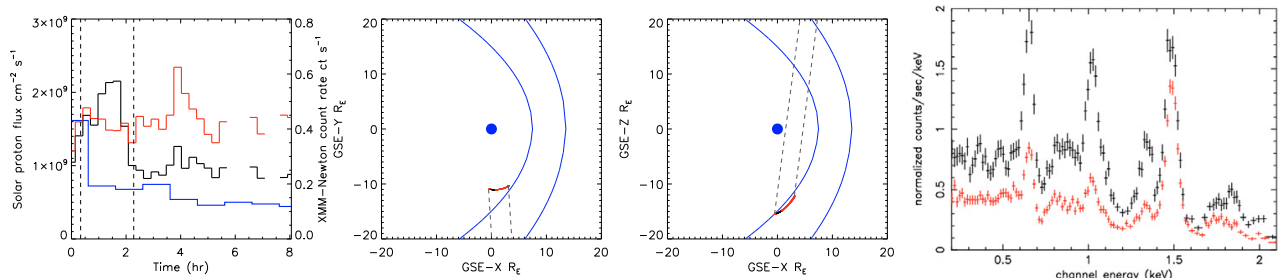

(a) Case: $\{1\}$, obsn. 0085150301
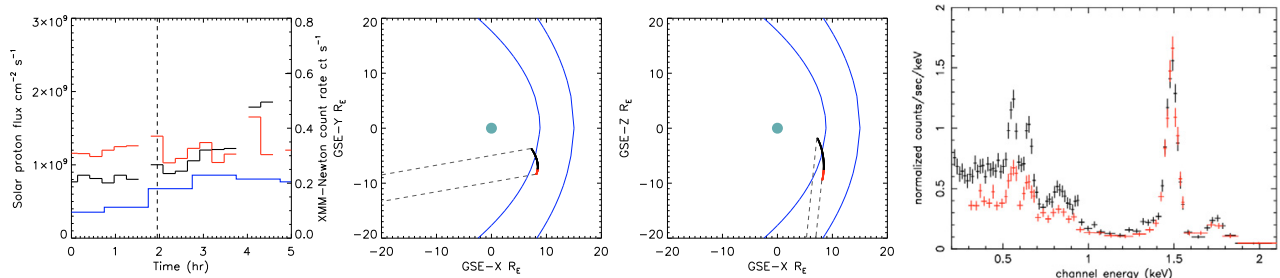

(b) Case: $\{3\}$, obsn. 0149630301
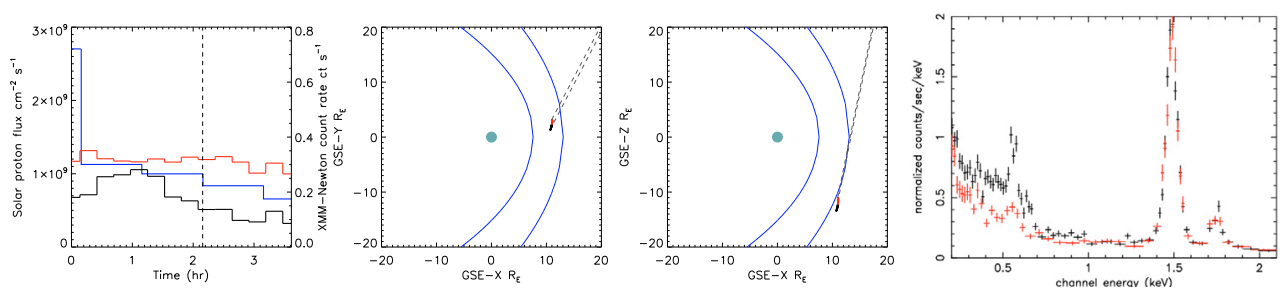

(c) Case: $\{4\}$, obsn. 0305920601
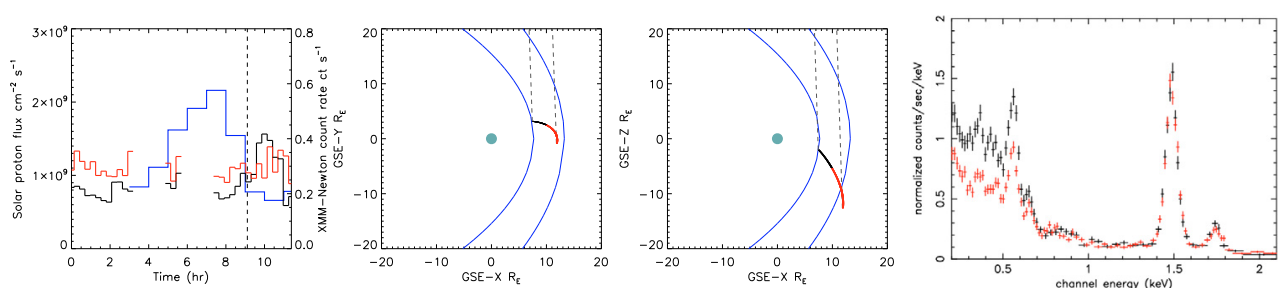

(d) Case: $\{5\}$, obsn. 0150680101
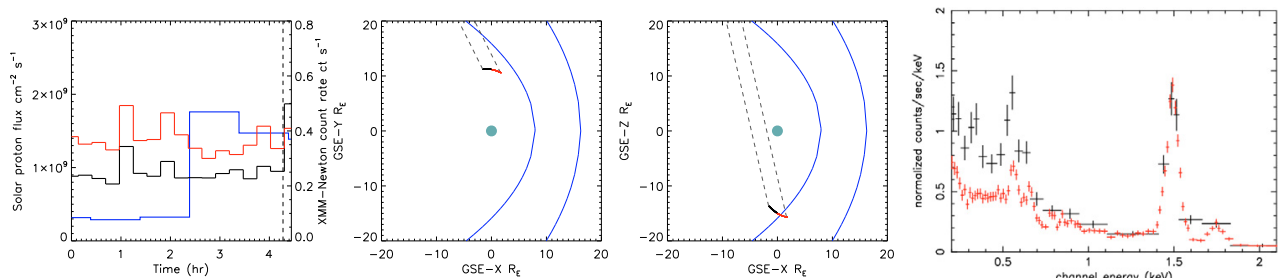

(e) Case: $\{14\}$, obsn. 0136000101

Fig. 12. XMM-Newton lightcurves, orbital positions and spectra for example cases of SWCX-enhancement. In the first panel for each case, the blue line represents the solar proton flux during each observation, plotted including the expected one hour delay and the vertical lines indicate the start or stop times for the division of the events into SWCX-enhanced and non SWCX-enhanced periods. Black and red represent the line and continuum band lightcurves respectively, similarly for the spectra shown in the last panel. The second and third panels show XMM-Newton in orbit around the Earth in the GSE $X-Y$ and $X-Z$ planes. The positive $x$-axis is directed towards the Sun and the positive $y$-axis is directed in the direction of the orbit of XMM-Newton.

solar proton flux could be correlated with the rise in the line band during the latter part of the observation. XMM-Newton was observing through the flanks of the magnetosheath throughout. The spectral case for SWCX-enhancement however is not as strong as for other cases.

- Case: $\{10\} 0070340501$

This observation is quite possibly affected by SWCX but has very few bins left after GTI-filtering and so we are reluctant to make any strong conclusions regarding the diagnosis. Emission lines of OVII, OVIII and some carbon are seen for the suspected enhanced period. The solar proton flux for this observation showed a peak that could correspond to the peak seen in the line band counts, although the increase in counts is seen directly after a filtered period of the lightcurve.

- Case: $\{17\} 0101440101$

This observation has very high levels of residual soft proton contamination and has been heavily GTI-filtered, but the spectra for the SWCX period show an enhancement below $0.7 \mathrm{keV}$ suggestive of SWCX-lines without any increase in flux for either the enhanced and non-enhanced 

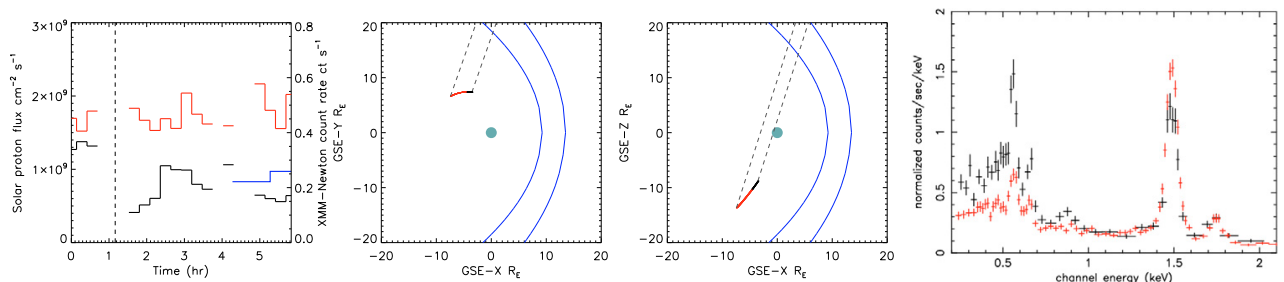

(a) Case: $\{2\}$, obsn. 0093552701
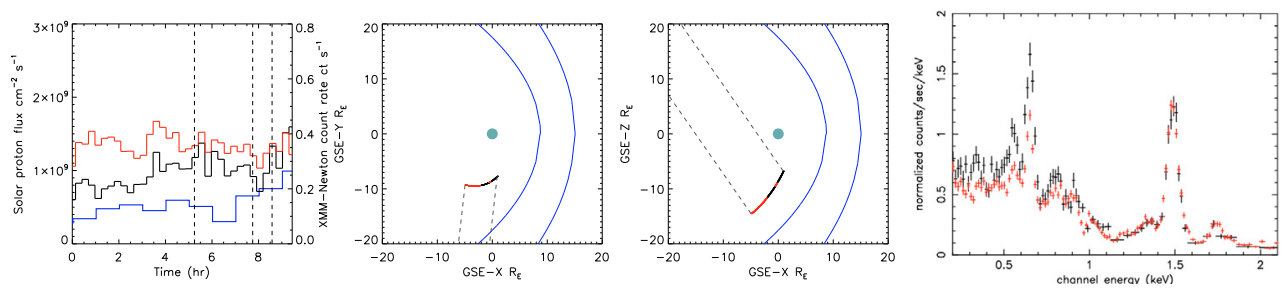

(b) Case: $\{6\}$, obsn. 0101040301
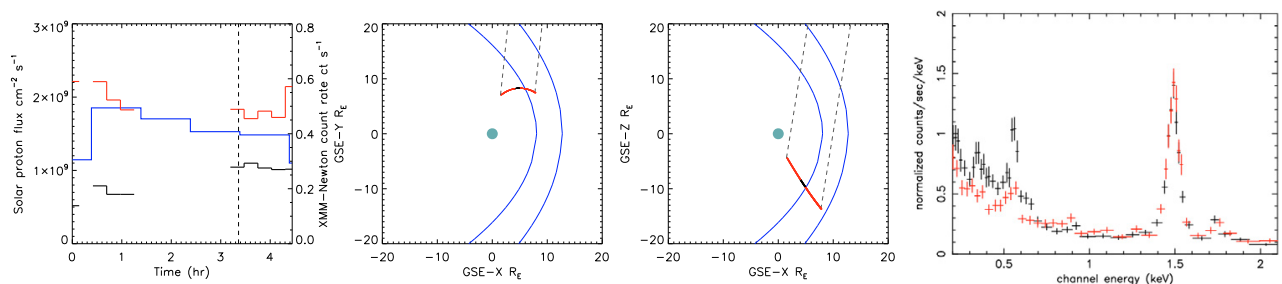

(c) Case: $\{10\}$, obsn. 0070340501
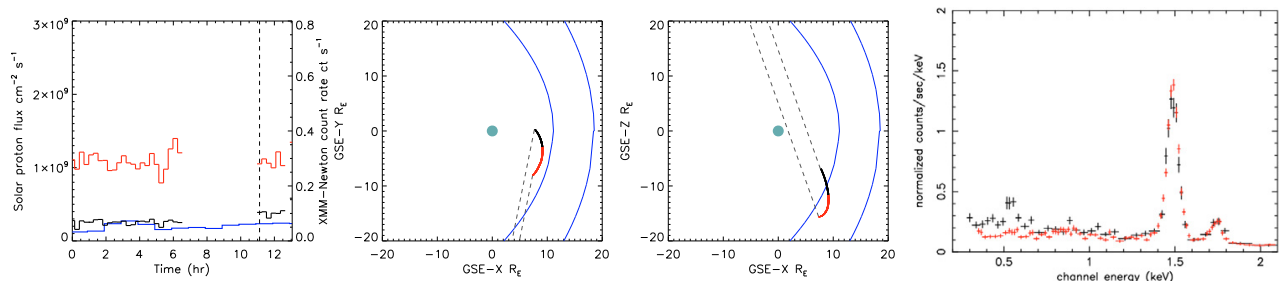

(d) Case: $\{17\}$, obsn. 0101440101
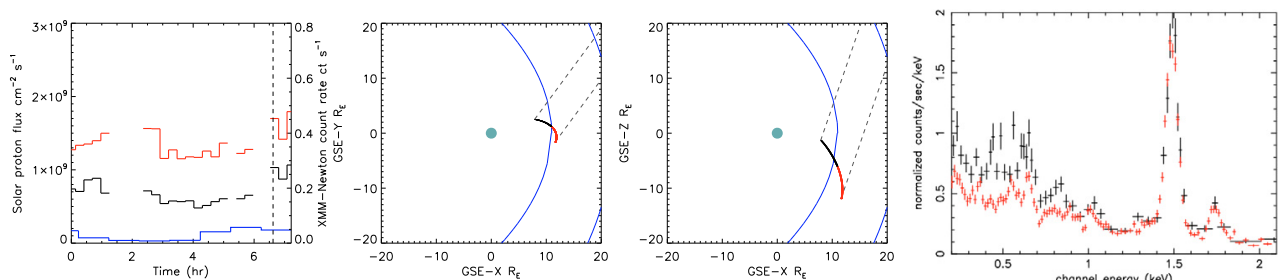

(e) Case: $\{25\}$, obsn. 0164560701
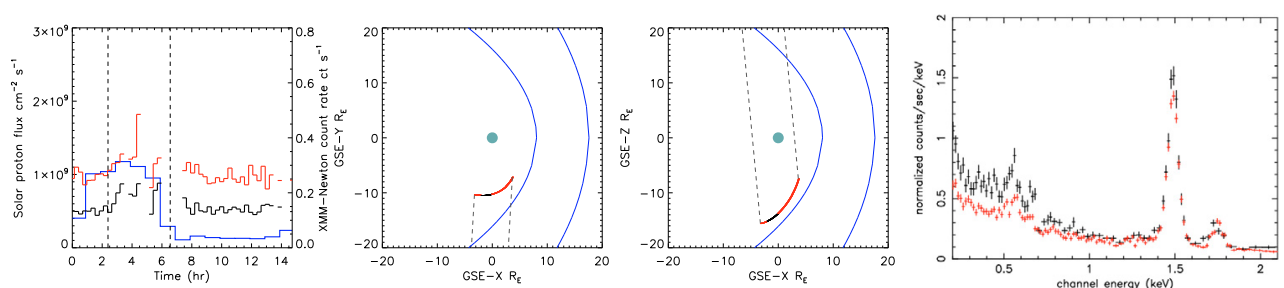

(f) Case: $\{28\}$, obsn. 0106460101

Fig. 13. Lightcurves, orbital positions and spectra for suspected cases of weak or dubious SWCX-enhancement, arranged in panels as for the strong cases of SWCX-enhancement.

periods above this energy. The enhanced period was taken after $12 \mathrm{~h}$. The $R_{\chi}$ of 1.56 is quite high. No correlation between the line band lightcurve and solar proton flux is seen, although XMM-Newton is positioned on the sub-solar side of the magnetosheath throughout the observation. For the enhancement period both the OVII and OVIII become apparent 
and there is possibly some increase in flux around the carbon lines below $0.5 \mathrm{keV}$.

\section{- Case: $\{25\} 0164560701$}

This observation is contaminated by residual soft protons. It does not have a perfect fit to the count rate histogram from the ESAS software and not a particularly high $R_{\chi}$ of 1.53 . The spectrum for the enhanced period shows very low level statistics although there is evidence of enhancement around the oxygen and carbon lines. The spectral split was taken at a time of seven hours. The observation was conducted in July when XMM-Newton was in the nose of the magnetosheath.

- Case: $\{28\} 0106460101$

This observation was taken in November when XMM-Newton was looking though the flanks of the magnetosheath. There is possibly some correlation between the line band lightcurve and the solar proton flux. The period for possible SWCX-enhancement was chosen to be between approximately two and seven hours. OVII, OVIII and some carbon enhancement is seen.

\section{Conclusions and further work}

We have presented a selection of results from a preliminary sample of approximately 170 XMM-Newton observations that have been tested for oxygen line variability as compared to a continuum band that is not expected to vary. Our primary measure of the likelihood of SWCX-enhancement is found through the lack of correlation between a count rate for the prime SWCX indicators, namely the band representing the OVII and OVIII major emission lines, and the count rate for a band that is SWCX-enhanced emission free. SWCX-enhanced observations showed both a high $\chi_{\mu}^{2}$ and $R_{\chi}$ value. In the event of soft proton contamination, correlation between the count rates from both bands is still expected. The level of anti-correlation is assessed using the metric of the $\chi_{\mu}^{2}$ from the relationship between a band representing the SWCX indicators and a band representing the emission line free continuum. We have demonstrated that the method has been successful in identifying cases of SWCX from a medium sized sample, providing careful consideration of various other diagnostic tests are taken into account. In addition, our method was also able to identify all but one of the control observations with previously identified SWCX that had been placed in the sample, within the top $35 \chi_{\mu}^{2}$-ranked results.

Our strongest case of SWCX-enhancement, with the richest spectrum showing many emission lines, gave the highest level of the $\chi_{\mu}^{2}$ value and presented a high $R_{\chi}$. This observation was contaminated by residual soft protons, although the resultant spectrum, between the non and SWCX-enhanced periods, was able to show many emission lines corresponding to highly charged ion species.

Cases which we believe present the best evidence of SWCX-enhancement exhibit enhanced spectral features such as emission lines of OVII and OVIII, along with lower energy lines probably associated with carbon. Two of the strongest cases offer emission lines that may be attributed to NeIX and MgXI. The pattern of observed emission lines depends on the exact composition of the solar wind at $1 \mathrm{AU}$ at the time of the observation.

We have shown that several of the cases where SWCX-enhancements are suspected occurred during periods when XMM-Newton was found to be on the sub-solar side of the magnetosheath. The line of sight of the telescope in these cases intersects the area of the magnetosheath of brightest expected solar wind-induced geocoronal X-ray flux. The SWCX-enhancement seen for other cases, when XMM-Newton was found to have a line of sight which intersects the magnetosheath through the weaker areas of X-ray flux, is probably due to a non-geocoronal origin, such as a CME density enhancement passing through the heliosphere.

In Paper II we will present results from the processing of a larger sample of observations to identify additional observations with SWCX-enhancement, extending our analysis to include data from the PN camera. We expect to find approximately 2000 observations of XMM-Newton affected by temporally variable SWCX, based on frequency of detections from the sample described in this paper. However, the number of detections is dependent on solar activity and therefore may vary considerably from the number stated. Using a larger sample, we aim to refine the identification of SWCX-enhancement, using the relationship between $\chi_{\mu}^{2}$ and $R_{\chi}$.

We will include an improved algorithm to remove sources in the field, reducing the danger of residual source contamination. We will investigate for any trends within the results that are related to the solar cycle and the position angle and orientation of XMM-Newton during each observation. This will help us to constrain the conditions when XMM-Newton is most likely to experience SWCX, whether that be due to the satellite's position or viewing geometry. We will also explore multiple pointings of the same target to consider variations between observations. We will investigate the observed SWCX line ratios and what this implies for the composition of the solar wind.

Acknowledgements. We would like to thank Simon Vaughan for helpful discussions regarding timing analysis and statistics and Kip Kuntz for similarly helpful discussions and advice. We would also like to thank the referee for his insightful comments and suggestions. This work has been funded by the Science and Technology Facilities Council, UK.

\section{References}

Branduardi-Raymont, G., Bhardwaj, A., Elsner, R. F., et al. 2007, A\&A, 463, 761

Collier, M. R., Moore, T. E., Snowden, S. L., \& Kuntz, K. D. 2005, Adv. Space Res., 35, 2157

Cox, D. P. 1998, in The Local Bubble and Beyond (Berlin: Springer)

Cravens, T. E. 1997, Geophys. Res. Lett., 24, 105

Cravens, T. E. 2000, ApJ, 532, L153

Cravens, T. E., Robertson, I. P., \& Snowden, S. L. 2001, J. Geophys. Res., 106, 24883

De Luca, A., \& Molendi, S. 2004, A\&A, 419, 837

Dennerl, K. 2002, A\&A, 394, 1119

Fujimoto, R., Mitsuda, K., Mccammon, D., et al. 2007, PASJ, 59, 133

Greenwood, J. B., Williams, I. D., Smith, S. J., \& Chutjian, A. 2000, ApJ, 533, L175

Hodges, R. R. J. 1994, J. Geophys. Res., 99, 23229

Jansen, F., Lumb, D., Altieri, B., et al. 2001, A\&A, 365, L1

Khan, H., \& Cowley, S. W. H. 1999, Ann. Geophys., 17, 1306

Koutroumpa, D., Acero, F., Lallement, R., Ballet, J., \& Kharchenko, V. 2007, A\&A, 475, 901

Kuntz, K. D., \& Snowden, S. L. 2008, A\&A, 478, 575

Robertson, I. P., \& Cravens, T. E. 2003a, J. Geophys. Res., Space Phys., 108, 8031

Robertson, I. P., \& Cravens, T. E. 2003b, Geophys. Res. Lett., 30, 080000

Robertson, I. P., Collier, M. R., Cravens, T. E., \& Fok, M.-C. 2006, J. Geophys. Res., Space Phys., 111, 12105

Snowden, S. L., Freyberg, M. J., Plucinsky, P. P., et al. 1995, ApJ, 454, 643

Snowden, S. L., Collier, M. R., \& Kuntz, K. D. 2004, ApJ, 610, 1182

Snowden, S. L., Mushotzky, R. F., Kuntz, K. D., \& Davis, D. S. 2008, A\&A, 478,615

Strüder, L., Briel, U., Dennerl, K., et al. 2001, A\&A, 365, L18

Turner, M. J. L., Abbey, A., Arnaud, M., et al. 2001, A\&A, 365, L27

Wang, X., Wurz, P., Bochsler, P., et al. 2005, in Coronal and Stellar Mass Ejections, ed. K. Dere, J. Wang, \& Y. Yan, IAU Symp., 226, 409

Wargelin, B. J., Markevitch, M., Juda, M., et al. 2004, ApJ, 607, 596

Weimer, D. R., Ober, D. M., Maynard, N. C., et al. 2003, J. Geophys. Res., Space Phys., 108, 1026 